\title{
The Euclid Near Infrared Spectro-Photometer (NISP) instrument and science
}

\author{
Stefano Dusini ${ }^{* a}$, Federico Fornari ${ }^{\dagger * b}$, Francesco Giacomini ${ }^{* c}$, Fulvio Laudisio ${ }^{* d}$, \\ Nicoletta Mauri $^{* b}$, Laura Patrizii ${ }^{* e}$, Chiara Sirignano*d ${ }^{*}$ Gabriele Sirri ${ }^{* e}$, Luca \\ Stanco ${ }^{* a}$, Matteo Tenti ${ }^{* f}$ and Luca Valenziano*e \\ ${ }^{a}$ INFN Padova \\ ${ }^{b}$ Department of Physics and Astronomy, University of Bologna, and INFN Bologna \\ ${ }^{c} \mathrm{CNAF}$ \\ ${ }^{d}$ Department of Physics and Astronomy, University of Padova, and INFN Padova \\ ${ }^{e}$ INFN Bologna \\ ${ }^{f}$ INFN-CNAF \\ E-mail: federico.fornari@bo.infn.it
}

\begin{abstract}
Euclid is an ESA mission designed to understand the nature of the Dark Energy responsible for the accelerated expansion of the Universe and constrain the nature of Dark Matter. These goals will be achieved by investigating the distance-redshift relationship and the evolution of cosmic structures. Euclid will be equipped with a $1.2 \mathrm{~m}$ diameter $\mathrm{SiC}$ mirror telescope feeding 2 instruments: the visible imager (VIS) and the Near-Infrared Spectro-Photometer (NISP). In this paper Euclid's observation probes and main aims are recalled, and the NISP instrument and expected performances are presented.
\end{abstract}

The European Physical Society Conference on High Energy Physics

5-12 July

Venice, Italy

\footnotetext{
${ }^{*}$ On behalf of the Euclid Consortium.

${ }^{\dagger}$ Speaker.
} 


\section{Introduction}

The Euclid mission will map the geometry of the dark Universe by investigating the distanceredshift relationship and the evolution of cosmic structures. The $1.2 \mathrm{~m}$ Korsch telescope will observe $15000 \mathrm{deg}^{2}$ of the sky from the Sun-Earth L2 Lagrangian point (excluding galactic and ecliptic planes). The telescope directs the light to two instruments via a dichroic filter in the exit pupil: the visual imager (VIS, 550-900 $\mathrm{nm}$ ) and the near-infrared spectrometer and photometer (NISP, Figure 1). Both instruments observe simultaneously a large field of view $\left(0.53 \mathrm{deg}^{2}\right)$. The system design is optimized for a sky survey in a step-and-stare tiling mode. The Euclid spacecraft will be launched in 2021 from the Guiana Space Centre, Kourou, on board a Soyuz rocket. The survey will last 6.25 years. The science data from the spacecraft will be received on ground at a rate of $850 \mathrm{Gbit} / \mathrm{day}$ over a daily pass time of 4 hours.
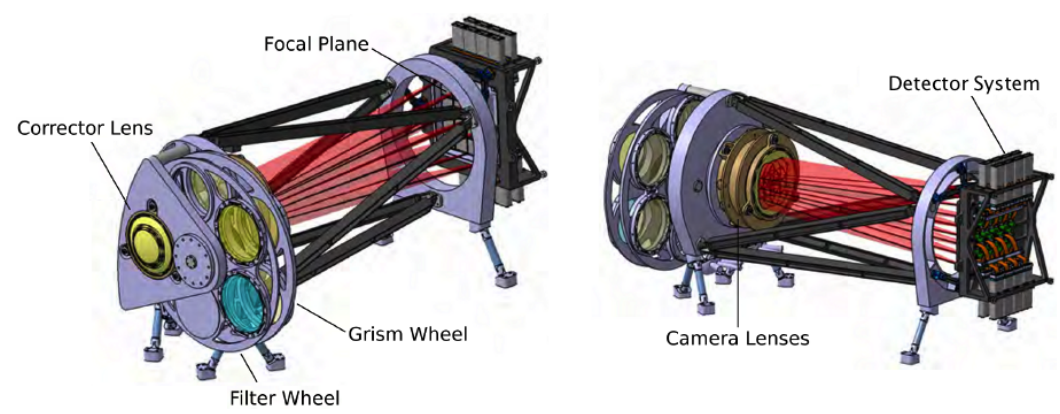

Figure 1: The NISP instrument with its subsystems.

Euclid's primary goal is to study the accelerated expansion of the Universe and the data collected by the mission will provide severe constraints on related theories. Two cosmological probes will be used:

1. Galaxy Clustering: by measuring spectroscopic redshifts 3D distribution of galaxies can be reconstructed. The Baryon Acoustic Oscillation model predicts the presence of overdensities in the distribution of galaxies at a relative distance related to the sound horizon of the perturbations generated in the primordial baryon-photon plasma at the recombination epoch $(z \simeq 1100)$. The BAO peak represents a standard ruler for the measurement of the expansion of the Universe because it is constant with time in a comoving reference frame; therefore, its evolution provides information about the expansion.

2. Weak Lensing: by measuring the correlation between the photometric deformation of the shape of galaxies, the distribution of ordinary and dark matter can be reconstructed, while the measurement of the photometric redshift of shape-distorted galaxies allows to trace back the information on their evolution.

In Table 1 the scientific specifications for weak lensing and galaxy clustering observations are summarized. Euclid will also allow to determine the total neutrino mass with high accuracy. The neutrino mass reflects on the process of cosmic structure formation since lighter neutrinos have more chances to travel longer distances, hence slightly weakening structure formation in the early 


\begin{tabular}{|c|c|c|c|c|c|}
\hline \multicolumn{3}{|c|}{ Galaxy Clustering } & \multicolumn{3}{|c|}{ Weak Lensing } \\
\hline $\begin{array}{l}\text { Spectroscopic } \\
\text { redshift accuracy }\end{array}$ & $\sigma_{z}<0.001(1+z)$ & $\begin{array}{l}0.7<z<1.8 \\
\text { with } 1700 \text { galaxies } / \mathrm{deg}^{2}\end{array}$ & $\begin{array}{l}\text { Photometric } \\
\text { redshift accuracy }\end{array}$ & $\sigma_{z}<0.05(1+z)$ & $\begin{array}{l}0<z<1.2 \\
\text { with } 30 \text { galaxies/arcmin }\end{array}$ \\
\hline
\end{tabular}

Table 1: Scientific specifications for weak lensing and galaxy clustering observations in Euclid [1].

Universe. A more or less intense suppression effect can be observed by Euclid in the matter power spectrum over a wide range of scales and in particular at small scales. The Euclid sensitivity to the sum of neutrino masses $\left(\Delta m_{v} \lesssim 0.05 \mathrm{eV}\right)$ will provide crucial information on the absolute scale, not accessible even to future laboratory experiments, and could allow the two mass hierarchies to be distinguished. Besides, Euclid will be able to improve the accuracy on the cosmological parameters describing the validity of General Relativity and the non-Gaussianity correction in primordial density fluctuations.

\section{The NISP Instrument}

The NISP Instrument operates as near-infrared spectrometer and photometer. In the photometer mode the instrument images the telescope light in the 920-2000 $\mathrm{nm}$ wavelength range, with a spatial resolution of 0.3 arcsec per pixel. In the spectrometer mode the light of the observed target is dispersed by grisms covering the wavelength range of 1.1-2 $\mu \mathrm{m}$. The NISP, with a mass of 155 $\mathrm{kg}$, will be placed in a box of $1 \times 0.5 \times 0.6 \mathrm{~m}^{3}$ and will produce $240 \mathrm{GBit}$ of data per day. It comprises:

- two filter wheels, one hosting the optical filters required for photometric observation (in Y, J and $\mathrm{H}$ bands) and the grisms allowing slitless spectroscopic observations.

- the focal plane, equipped with a $4 \times 4$ array of $\mathrm{HgCdTe} 2048 \times 2048$ pixel detectors and the Sensor Chip System [2]. The operating temperature of the detectors is lower than $100 \mathrm{~K}$ while each individual readout electronic (ASIC for digitization) operates at around $140 \mathrm{~K}$ in order to ensure low thermal noise.

- the calibration unit, providing near-uniform illumination in several wavelength channels of the detector plane, ensuring a stable flat sensor calibration source.

- the warm electronics system, composed of 2 units:

- two Data Processing Units (DPU), each allowing synchronous acquisition of 8 detectors, on-board data compression, pre-processing and transfer to the spacecraft mass memory. Each DPU contains 8 Detector Control Unit boards, each connected to a Sensor Chip System.

- the Instrument Control Unit (ICU) provided with a cold redundant unit, exchanges telemetry and telecommand data with the DPU and includes control electronics for filter wheels and calibration unit. It is also responsible for temperature sensors monitoring/heaters powering and it is interfaced with the satellite via a MIL-STD-1553 bus. 
The interface with the DPU is also based on a 1553 bus. The ICU Application software (ASW) is devoted to manage the satellite/platform interface, the ICU/DPU interface and all the functionalities related to instrument commanding. The ICU uses a space qualified version of RTEMS real-time operating system [3].

The warm electronics is located in the service module of the spacecraft at ambient temperature. The NISP functional architecture is shown in Figure 2. A more detailed description can be found in [4].

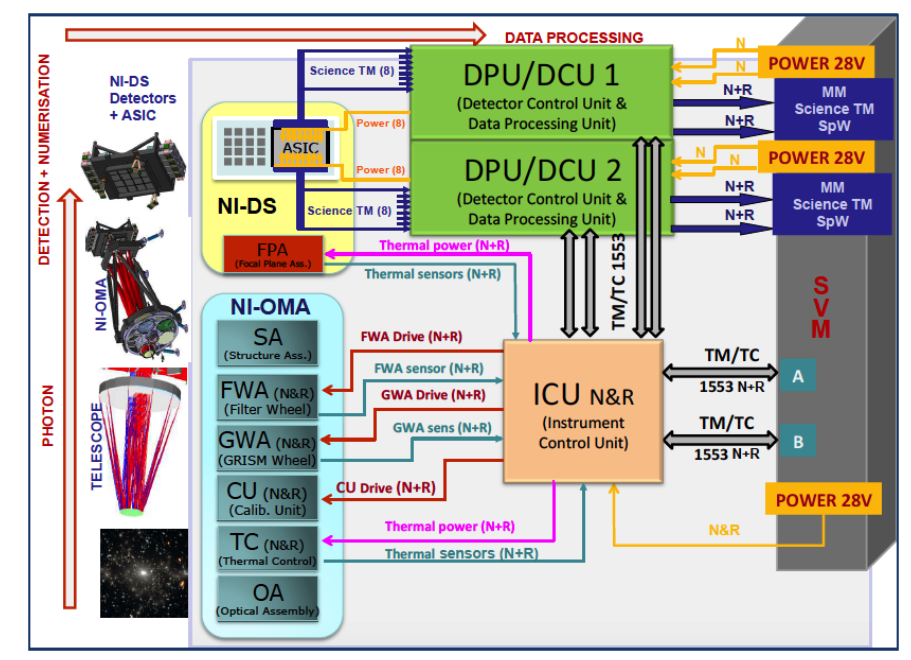

Figure 2: Overview of NISP functional architecture.

\section{NISP operation and performance}

The NISP observing sequence consists of $565 \mathrm{~s}$ for spectroscopy observation, 121, 116 and $81 \mathrm{~s}$ for the Y, J, H photometric bands, respectively (Figure 3). The wheels activate between each observation to set the instrument in the proper configuration. At the end of each sequence a slew is performed in order to change the field of view. For the spectroscopic observation, the minimum flux for the detection is $2 \times 10^{-16} \mathrm{erg} \cdot \mathrm{s}^{-1} \cdot \mathrm{cm}^{-2}$, corresponding to $\sim 3.5 \sigma \mathrm{SNR}$. In addition, photometric detection limit in NIR wavelengths for $5 \sigma$ SNR point sources is of $24 \mathrm{AB}$ magnitude [4]. The number of expected observed galaxies is $2.5 \times 10^{7}$ and $1.5 \times 10^{9}$ for the spectroscopic and the photometric sample, respectively.

\section{Conclusions}

Different NISP models developed, namely: the Structure and Thermal Model (STM) to validate the design of the optical assembly and the detector system. This model has been already successfully tested. The NISP Engineering Model (EM) will be built to fully qualify the instrument functional behaviour. Then a NISP Avionic Model consisting in a subset of the NISP EM equipped with DPU and ICU qualification models will be realized. Finally, the NISP Flight Model will be delivered to ESA. 


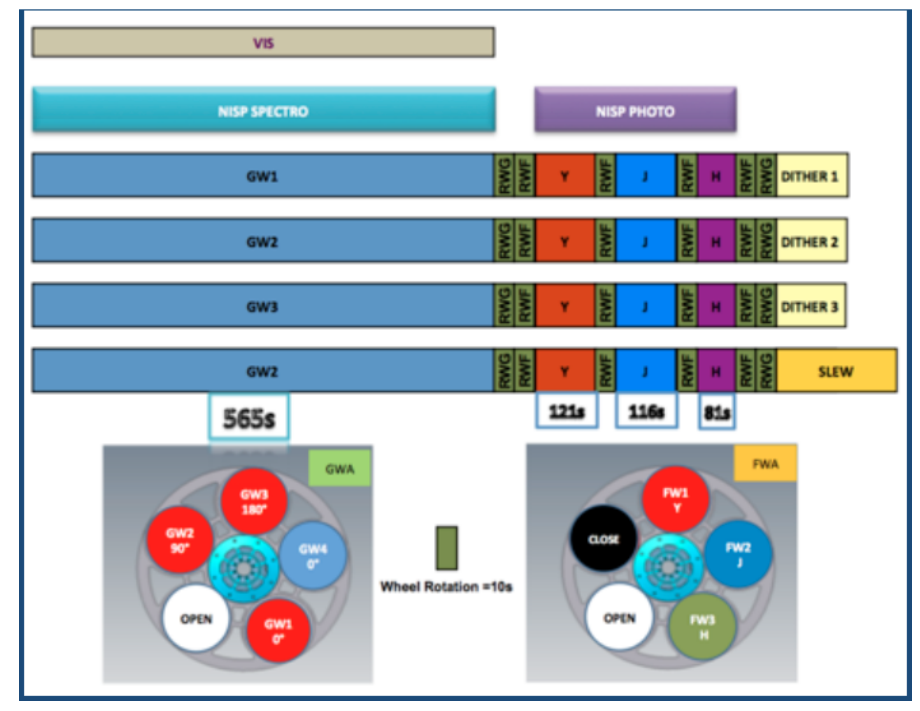

Figure 3: The NISP observing sequence.

\section{Acknowledgements}

We acknowledge financial contribution from the agreement ASI/INAF I/023/12/1.

\section{References}

[1] R. Laureijs et al., Euclid Definition Study Report, arXiv:1110.3193.

[2] J. W. Beletic et al. Proc. SPIE 7021 (2008).

[3] S. Ligori et al. Proc. SPIE 9904 (2016).

[4] T. Maciaszek et al. Proc. SPIE 9904 (2016) . 